J. AMER. SOC. HorT. SCI. 115(3):348-356. 1990.

\title{
Strain and Rootstock Effects on Spur Characteristics and Yield of 'Delicious' Apple Strains
}

\author{
Ian J. Warrington ${ }^{1}$, David C. Ferree, and James R. Schupp ${ }^{2}$ \\ Department of Horticulture, The Ohio Agricultural Research and Development Center, The Ohio \\ State University, Wooster, $\mathrm{OH} 44691$
}

Frank G. Dennis, Jr.

Department of Horticulture, Michigan State University, East Lansing, MI 48824

Tara A. Baugher

West Virginia University Experiment Farm, Box 303, Kearneysville, WV 25430

Additional index words. Malus domestica, leaf area, leaf number, yield efficiency, spur density

\begin{abstract}
The characteristics of l-year-old vegetative spurs growing on 2-year-old branches were measured on 28 'Delicious' apple (Malus domestica Borkh.) strains growing on M.7 rootstocks at Clarksville, Mich., and on 23 strains of 'Delicious' on M.7a rootstocks at Kearneysville, W.Va. Spur-type strains typically had densities >20 to 21 spurs/m, and high spur leaf numbers, leaf areas per spur, leaf areas per leaf, and terminal bud diameters, whereas values for standard strains were generally lower. However, for most spur quality characteristics, there was a continuous range of values between the extremes rather than any distinct grouping into either spur or standard type. At both sites, spur density was significantly and positively correlated with yield efficiency. In a related study, the spur characteristics of 'Starkspur Supreme' were measured on nine rootstocks: M.7 EMLA, M.9 EMLA, M.26 EMLA, M.27 EMLA, M.9, MAC 9, MAC 24, OAR 1, and Ottawa 3. Spur leaf number and spur leaf area were both high with vigorous rootstocks, whereas spur density was low. The rootstocks MAC 9, M.9, and M.9 EMLA had the highest yield efficiencies.
\end{abstract}

A feature of red apple cultivars in general, and of 'Delicious' in particular, is the availability of many different strains from commercial nurseries (Tukey and Ballard, 1969a). Most 'Delicious' strains have been selected primarily either for some specific fruit characteristic (mainly color and shape) or for a particular type of growth and related spur-bearing habit that is predominantly either a spur type or standard form (Fisher and Ketchie, 1981; Tukey and Ballard, 1969b). However, while fruit characteristics are well known and definable (Crassweller et al., 1985; Fisher and Ketchie, 1981; Ingle, 1972; Lord et al., 1980), few data are available on the yield performance of the different strains or on the relative performance of strains within and among apple-producing regions (Dozier et al., 1984; Ferree et al., 1975; Ketchie, 1984, 1987; Lord et al., 1980; Rom and Ferree, 1984). Therefore, the selection of the most appropriate and highest-yielding strains for a particular region is not possible unless either comparative regional trials are carried out (Ketchie, 1987) or indices can be identified that would allow a prediction to be made of relative performance.

An obvious difference in the growth characteristics of various 'Delicious' strains is the degree of spur-bearing habit. Spur leaves are critically important for fruit development (Ferree and Palmer, 1982), and differences in spur habit and form may, therefore, influence fruit yield and the distribution of fruit on a tree. Before bloom, spur leaves constitute the major leaf area on a

Received for publication 8 June 1989. Salaries and research support provided by state and federal funds appropriated to the Ohio Agricultural Research and Development Center, The Ohio State Univ. Journal Article no. 18-89. The cost of publishing this paper was defrayed in part by the payment of page charges. Under postal regulations, this paper therefore must be hereby marked advertisement solely to indicate this fact.

${ }^{1}$ Present address: Plant Physiology Division, DSIR, Private Baa, Palmerston North, New Zealand.

${ }^{2}$ Present address: Highmoor Farm, Univ. of Maine, PO Box 178, Monmouth, ME 04259 . tree and provide the majority of photosynthate available for early fruit growth and development (Hansen, 1971). During fruit set and the cell division phase of fruit growth, spur leaves play a major role in determining fruit size, shape, and fruit calcium concentration (Ferree and Palmer, 1982). The removal of spur leaves before and during bloom reduces fruit set (Dalbro, 1966), while a certain minimum spur leaf area is necessary for flower bud formation (Harley et al., 1942).

Differences among apple cultivars for spur diameter, flower number, spur leaf number, area, and individual spur leaf size have been reported, and total spur leaf area or average spur leaf size has been related to tree productivity, yield efficiency, and yield variation (Rom and Ferree, 1984). The objective of this study was to evaluate these relationships on various commercially designated spur and standard 'Delicious' strains growing in two locations. In addition, the influence of rootstock on spur quality, tree size, and yield of 'Starkspur Supreme Delicious' was investigated on trees planted at the Ohio Agricultural Research and Development Center (OARDC), Wooster.

(Throughout the manuscript, the word 'Delicious' has been deleted from each strain name to avoid unnecessary repetition.)

\section{Materials and Methods}

Twenty-eight 'Delicious' strains were evaluated at the Michigan State Univ. Clarksville Horticultural Research Station (Table 1). The trees were established in 1980 in a planting 18 trees long and 21 rows wide, with each strain represented by one tree in each of six blocks. All of the strains studied were planted on M.7 EMLA rootstock. An equal number of trees on MM.111 EMLA rootstock were randomly distributed among the study trees, and four individual trees of 'Idared' were included in each block as pollenizers. Trees of similar age and size surrounded the planting. The within/between row spacing was $3.65 \times 5.5$ $\mathrm{m}$. The trees were part of a cooperative research program to 
Table 1. 'Delicious' strains studied, name of the supplying nursery, plant patent number, growth type, and location of study site.

\begin{tabular}{|c|c|c|c|c|}
\hline Strain & $\begin{array}{l}\text { Nursery } \\
\text { company }\end{array}$ & $\begin{array}{l}\text { U.S. plant } \\
\text { patent }\end{array}$ & $\begin{array}{l}\text { Growth } \\
\text { type }\end{array}$ & $\begin{array}{l}\text { Study } \\
\text { site }\end{array}$ \\
\hline Ace & Columbia Basin* & & Spur & Michigan \\
\hline ACN 501 & Adams County ${ }^{\mathrm{y}}$ & & Spur & West Virginia \\
\hline Aomori & Stark Bros. ${ }^{\mathrm{x}}$ & & Standard & Michigan, West Virginia \\
\hline Apex & Columbia Basin & & Spur & Michigan, West Virginia \\
\hline Atwood & Yakima Valley $^{\mathrm{w}}$ & & Spur & Michigan \\
\hline August Red & Heath $^{\mathrm{v}}$ & & Standard & Michigan \\
\hline Cascade Spur & Carlton $^{\mathrm{u}}$ & 4801 & Spur & West Virginia \\
\hline Classic Red & $\mathrm{C} \& \mathrm{O}^{\mathrm{t}}$ & 3182 & Standard & Michigan, West Virginia \\
\hline Early Red One & Van Well ${ }^{\mathrm{s}}$ & 3556 & Standard & Michigan \\
\hline Hardi-Brite Spur & Carlton & & Spur & Michigan \\
\hline Hardispur & Carlton & & spur & Michigan \\
\hline Hi-Early & Heath & & Standard & Michigan \\
\hline Imperial & Hilltop & 1805 & Standard & Michigan, West Virginia \\
\hline Improved Ryanred & Carlton & & Standard & Michigan, West Virginia \\
\hline Improved Ryan Spur & Carlton & & Spur & Michigan \\
\hline Nured Royal & $\mathrm{C} \& \mathrm{O}$ & & Standard & Michigan, West Virginia \\
\hline Redchief (Campbell) & Hilltop & 3578 & Spur & Michigan, West Virginia \\
\hline Red King Oreg & Van Well & 2816 & Spur & Michigan \\
\hline Red Prince & Hilltop & 2285 & Standard & West Virginia \\
\hline Redspur & $\mathrm{C} \& \mathrm{O}$ & 1822 & Spur & Michigan, West Virginia \\
\hline Rose Red & McCormick $^{\mathrm{q}}$ & 3485 & Standard & Michigan \\
\hline Ryanred Spur & Carlton & & Spur & West Virginia \\
\hline Sharp Red & Van Well & & Standard & Michigan, West Virginia \\
\hline Silver Spur & Bountiful Ridge ${ }^{p}$ & & Spur & Michigan \\
\hline Spured Royal & $\mathrm{C} \& \mathrm{O}$ & 3864 & Spur & Michigan \\
\hline Starking (Mood) & Stark Bros. & & Standard & Michigan \\
\hline Starkrimson (Bisbee) & Stark Bros. & 1565 & Spur & Michigan, West Virginia \\
\hline Starkspur Supreme & Stark Bros. & 3541 & Spur & Michigan, West Virginia \\
\hline Starkspur Ultrared & Stark Bros. & 3557 & Spur & Michigan, West Virginia \\
\hline Starkspur UltraStripe & Stark Bros. & 5472 & Spur & West Virginia \\
\hline $\begin{array}{l}\text { Sturdeespur } \\
\text { (Miller Sturdeespur) }\end{array}$ & Hilltop & 2433 & Spur & Michigan, West Virginia \\
\hline Topred & $\mathrm{C} \& \mathrm{O}$ & 1916 & Standard & Michigan, West Virginia \\
\hline Topspur & $\mathrm{C} \& \mathrm{O}$ & 5334 & Spur & West Virginia \\
\hline Triple Red & Adams County & & Standard & West Virginia \\
\hline Wellspur & Van Well & & Spur & Michigan, West Virginia \\
\hline
\end{tabular}

${ }^{7}$ Columbia Basin, P.O. Box 458, Quincy, WA 98848.

${ }^{y}$ Adams County Nursery, Inc., P.O. Box 108, Aspers, PA 17304.

${ }^{\mathrm{x}}$ Stark Bros. Nurseries and Orchards Co., Louisiana, MO 63353.

wakima Valley Nursery, 6461 W. Powerhouse Rd., Yakima, WA 98908.

${ }^{\vee}$ Heath Nursery, Brewster, WA 98812.

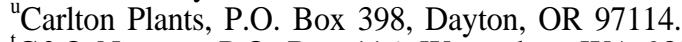

${ }^{t}$ C\&O Nursery, P.O. Box 116, Wenatchee, WA 98801-0122.

${ }^{\mathrm{s}}$ Van Well Nursery, P.O. Box 1339, Wenatchee, WA 98801.

${ }^{\mathrm{r}}$ Hilltop Orchards \& Nurseries, Inc., P.O. Box 578, CR 681, Hartford, MI 49057.

${ }^{\mathrm{q}}$ McCormick Fruit Tree Co., 6111-A Englewood Ave., Yakima, WA 98908.

${ }^{\mathrm{P}}$ Bountiful Ridge Nursery, Princess Anne, MD 21853.

evaluate 'Delicious strains at eight locations in the United States and in British Columbia (Ketchie, 1987).

Of the 23 strains at the West Virginia Univ. Experiment Farm at Kearneysville (Table 1), 15 were planted in Apr. 1981, six ('Starkspur', 'Ultrared', 'Starkrimson', 'Starkspur Supreme', 'Aomori', and 'Cascade Spur') in Apr. 1982, and three ('Starkspur UltraStripe', 'ACN 501', and 'Redchief) in Mar. 1983. They were arranged in a single planting 45 trees long and 11 rows wide, with. each strain represented in two blocks of five trees randomly distributed within the planting. 'Topspur' was planted in only one block. A similar number of block of trees on rootstocks other than those on M.7a used for the study were randomly distributed throughout the planting. A tree of 'Golden Delicious'/M.26 was planted between the two blocks of five trees within each row. The within/between row spacing was 4.9 $\times 6.1 \mathrm{~m}$.

Trees in both plantings were trained to a central leader and lateral branches were headed. Other pruning and training were minimal, allowing the trees to grow as close to their natural form as possible. The fruit was not chemically thinned and hand thinning was minimal. Otherwise, standard cultural practices were followed.

The influence of rootstock on 'Delicious' performance was studied using trees in the North Central Regional Project (NC140) planted in 1980 at the OARDC Horticulture Unit 2 at Wooster, Ohio. 'Starkspur Supreme' (Paganelli selection) was budded onto the following rootstocks: Ottawa 3, M.7 EMLA, M.9 EMLA, M.26 EMLA, M.27 EMLA, M.9, MAC 9 (Mark), 
MAC 24, and OAR 1. Ten trees of each rootstock were randomly planted in a block 20 trees long and six rows wide at a spacing of $3.5 \times 5.5 \mathrm{~m}$. 'Macspur' and 'Starkspur Golden Delicious' were used as pollenizers.

All spur characteristics were measured on 1-year-old vegatative spurs graving on 2-year-old branches. Five spurs were removed, and leaf number, leaf area, leaf dry weight, and terminal bud diameter were measured. Spur density was determined by measuring the length of 2-year-old growth on five randomly selected branches and counting the spurs within that section.

Samples were collected at Clarksville on 17 June 1986, at Kearneysville on 23 Sept. 1986, and at Wooster on 13 Aug. 1986. Fruit were picked at the commercial harvest time in each region. At Clarksville and Wooster, harvests were made of individual trees, while at Kearneysville, values were recorded for the five-tree replicates.

\section{Results}

Density of spurs. The density of spurs on 2-year-old branches varied widely at both sites and both within and between spur and standard types. Within the commercially designated spur types at Clarksville (Table 2), spur density (all per meter length) varied from 24 in 'Starkspur Ultrared' to 34 in 'Hardi-Brite Spur'. At Kearneysville (Table 3), the range extended from 19 in 'Topspur' to 39 in 'Sturdeespur'. Among the standard types, spur density ranged from 22 in 'Improved Ryanred' to 14 in 'Sharp Red' at Clarksville and from 32 in 'Imperial' to 15 in 'Nured Royal' at Kearneysville.
Where the same strains were assessed at both sites, the ranking of strains, with respect 'to spur density, was similar, except for 'Starkrimson', which had a lower relative density at Kearneysville. With the exception of 'Starkrimson', 'Wellspur', and 'Nured Royal', spur density was consistently higher (by $13 \%$ on average) at Kearneysville than at Clarksville. At Kearneysville, only one or two strains, designated as spur or standard types, did not fit closely into the respective groups containing similar types. Spur density was greater for the standard strain 'Imperial' and less for the spur strain 'Topspur' than for other similar types.

At both sites, the spur-type strains generally had a spur density $>20$ to $21 \mathrm{spurs} / \mathrm{m}$, while the standard types had a lower density. However, the classification of strains into standard or spur types was only relative; there was a steady gradation of, spur density from the most to the least spur bearing, with no major separation on between types.

Leaf number per spur. The number of leaves per spur was generally higher for spur-type strains than for standard strains. At Kearneysville (Table 3), spur types ranged from 8.2 ('Redspur') to 6.9 ('Wellspur') and standard types from 6.9 ('Aomori') to 5.9 ('Sharp Red'). The same trend was apparent in the Clarksville data (Table 2). In strains common to both sites, leaf numbers per spur were similar.

Leaf area per spur. Leaf area/spur varied almost 2-fold between the highest- and lowest-ranking strains at each site. Although the ranking was not identical for those strains that were common to both sites, there were some consistent trends. For

Table 2. Characteristics of 1-year-old vegetative spurs from 28 'Delicious' apple strains growing at the Michigan State Univ. Clarksville Horticultural Research Station. ${ }^{\mathrm{Z}}$

\begin{tabular}{|c|c|c|c|c|c|c|c|}
\hline $\begin{array}{c}\text { Delicious } \\
\text { strain }\end{array}$ & Type & $\begin{array}{c}\text { Spur } \\
\text { density } \\
\text { (no./m) }\end{array}$ & $\begin{array}{c}\text { Leaf } \\
\text { no./spur }\end{array}$ & $\begin{array}{c}\text { Leaf area/spur } \\
\left(\mathrm{cm}^{2}\right)\end{array}$ & $\begin{array}{c}\text { Leaf area/leaf } \\
\left(\mathrm{cm}^{2}\right)\end{array}$ & $\begin{array}{c}\text { specific } \\
\text { leaf wt } \\
\left(\mathrm{mg} \cdot \mathrm{cm}^{-2}\right)\end{array}$ & $\begin{array}{c}\text { Bud } \\
\text { diam } \\
(\mathrm{mm}) \\
\end{array}$ \\
\hline Hardi-Brite Spur & Spur & 34 & 7.9 & 103 & 13.2 & 9.57 & 3.10 \\
\hline Improved Ryan Spur & Spur & 33 & 7.5 & 99 & 13.1 & 8.56 & 2.97 \\
\hline Red King Oregon Spur & Spur & 31 & 7.5 & 99 & 13.2 & 9.67 & 3.08 \\
\hline Starkrimson & Spur & 31 & 7.2 & 86 & 12.0 & 9.44 & 2.79 \\
\hline Spured Royal & Spur & 31 & 7.9 & 89 & 11.4 & 9.08 & 2.94 \\
\hline Redchief & Spur & 29 & 8.2 & 88 & 10.7 & 9.80 & 2.94 \\
\hline Hardispur & Spur & 29 & 7.5 & 96 & 12.9 & 9.66 & 2.88 \\
\hline Redspur & Spur & 29 & 7.9 & 88 & 11.1 & 9.33 & 2.85 \\
\hline Silverspur & Spur & 28 & 7.6 & 102 & 13.4 & 9.01 & 2.92 \\
\hline Ace & Spur & 28 & 8.0 & 109 & 13.8 & 8.95 & 3.09 \\
\hline Sturdeespur & Spur & 28 & 8.1 & 102 & 12.6 & 9.63 & 3.04 \\
\hline Apex & Spur & 28 & 7.8 & 98 & 12.6 & 9.25 & 3.02 \\
\hline $\begin{array}{l}\text { Apex } \\
\text { Starkspur Supreme }\end{array}$ & $\begin{array}{l}\text { Spur } \\
\text { Spur }\end{array}$ & 28 & 8.0 & 77 & 9.7 & 9.68 & 2.78 \\
\hline Wellspur & Spur & 27 & 8.0 & 96 & 12.0 & 9.37 & 2.91 \\
\hline Atwood & Spur & 25 & 8.1 & 92 & 11.3 & 9.33 & 2.97 \\
\hline Atwood & Spur & 25 & 8.7 & 110 & 12.6 & 9.22 & 2.98 \\
\hline Improved Ryanred & Standard & 22 & 6.7 & 65 & 9.6 & 9.41 & 2.69 \\
\hline Early Red One & Standard & 21 & 7.8 & 66 & 8.4 & 9.19 & 2.82 \\
\hline Imperial & Standard & 20 & 6.8 & 69 & 10.2 & 9.35 & 2.79 \\
\hline August Red & Standard & 18 & 7.6 & 73 & 9.8 & 8.60 & 2.65 \\
\hline Nured Royal & Standard & 18 & 7.2 & 95 & 13.1 & 8.53 & 2.95 \\
\hline Starking (Mood) & Standard & 18 & 7.2 & 79 & 11.0 & 9.17 & 2.80 \\
\hline Rose Red & Standard & 17 & 7.1 & 82 & 11.5 & 8.97 & 2.85 \\
\hline Classic Red & Standard & 16 & 7.5 & 84 & 11.4 & 9.14 & 2.81 \\
\hline Aomori & Standard & 15 & 7.1 & 67 & 9.5 & 9.50 & 2.68 \\
\hline Topred & Standard & 15 & 6.8 & 82 & 12.0 & 8.23 & 2.78 \\
\hline Hi-Early & Standard & 15 & 6.9 & 73 & 10.6 & 8.98 & 2.79 \\
\hline Sharp Red & Standard & 14 & 6.9 & 74 & 10.9 & 8.99 & 2.82 \\
\hline $\operatorname{LSD}(P<0.05)$ & & 6 & 0.8 & 16 & 1.7 & 0.72 & 0.22 \\
\hline
\end{tabular}

${ }^{\mathrm{z}}$ Data are means of six replications. 
Table 3. Characteristics of 1-year-old vegetative spurs from 23 'Delicious' apple strains growing at the West Virginia Univ. Experiment Farm, Kearneysville. ${ }^{\mathrm{Z}}$

\begin{tabular}{|c|c|c|c|c|c|c|c|}
\hline $\begin{array}{c}\text { Delicious } \\
\text { strain }\end{array}$ & Type & $\begin{array}{c}\text { Spur } \\
\text { density } \\
\text { (no./m) } \\
\end{array}$ & $\begin{array}{c}\text { Leaf } \\
\text { no./spur } \\
\end{array}$ & $\begin{array}{c}\text { Leaf area/spur } \\
\left(\mathrm{cm}^{2}\right)\end{array}$ & $\begin{array}{c}\text { Leaf area/leaf } \\
\left(\mathrm{cm}^{2}\right)\end{array}$ & $\begin{array}{c}\text { Specific } \\
\text { leaf wt } \\
\left(\mathrm{mg} \cdot \mathrm{cm}^{-2}\right)\end{array}$ & $\begin{array}{r}\text { Bud } \\
\text { diam } \\
(\mathrm{mm}) \\
\end{array}$ \\
\hline Sturdeespur & Spur & 39 & 7.5 & 79 & 10.5 & 11.0 & 3.80 \\
\hline Redchief & Spur & 38 & 7.6 & 88 & 11.6 & 11.4 & 3.78 \\
\hline Starkspur ultraStripe & Spur & 36 & 7.9 & 96 & 12.2 & 11.6 & 4.19 \\
\hline Redspur & Spur & 33 & 8.2 & 86 & 10.4 & 11.9 & 3.78 \\
\hline Ace & Spur & 33 & 8.0 & 88 & 10.8 & 11.8 & 4.20 \\
\hline Imperial & Standard & 32 & 6.4 & 61 & 9.4 & 11.3 & 3.96 \\
\hline Apex & Spur & 29 & 8.1 & 86 & 10.6 & 12.0 & 4.05 \\
\hline $\begin{array}{l}\text { Apex } \\
\text { Starkspur Supreme }\end{array}$ & Spur & 29 & 7.7 & 76 & 9.8 & 12.1 & 3.62 \\
\hline Cascade Spur & Spur & 29 & 8.0 & 95 & 11.8 & 12.2 & 4.11 \\
\hline ACN 501 & Spur & 29 & 7.6 & 105 & 13.7 & 11.6 & 4.18 \\
\hline Starkspur Ultrared & Spur & 28 & 7.7 & 88 & 11.4 & 12.0 & 3.91 \\
\hline Ryanred Spur & Spur & 27 & 7.6 & 95 & 12.5 & 11.9 & 4.04 \\
\hline Starkrimson & Spur & 26 & 7.2 & 90 & 12.4 & 11.4 & 3.97 \\
\hline Improved Ryanred & Standard & 25 & 6.8 & 57 & 8.4 & 11.7 & 3.71 \\
\hline Wellspur & Spur & 24 & 6.9 & 74 & 10.6 & 11.8 & 3.97 \\
\hline Triple Red & Standard & 20 & 6.0 & 67 & 11.2 & 10.7 & 3.81 \\
\hline Classic Red & Standard & 20 & 6.6 & 73 & 10.9 & 11.3 & 3.92 \\
\hline Red Prince & Standard & 19 & 6.1 & 71 & 11.5 & 11.3 & 3.81 \\
\hline Topspur & Spur & 19 & 7.0 & 76 & 10.8 & 12.1 & 3.73 \\
\hline Aomori & Standard & 18 & 6.9 & 81 & 11.6 & 11.5 & 3.93 \\
\hline Topred & Standard & 18 & 6.4 & 72 & 11.2 & 11.3 & 3.73 \\
\hline Sharp Red & Standard & 16 & 5.9 & 66 & 11.1 & 11.3 & 3.76 \\
\hline Nured Royal & Standard & 15 & 6.4 & 73 & 11.3 & 10.9 & 3.72 \\
\hline $\operatorname{LSD}(P<0.05)$ & & 9 & 0.6 & 11 & 1.3 & $\ldots y$ & 0.23 \\
\hline
\end{tabular}

${ }^{\mathrm{z}}$ Data are means of two blocks, each containing five trees.

${ }^{\mathrm{y}}$ Pooled weight values; no statistical analysis possible.

example, 'Starkspur Ultrared' had a large leaf area/spur at both sites; 'Improved Ryan Red', 'Imperial', and 'Sharp Red' had small areas; and 'Redchief', 'Redspur', 'Apex', and 'Starkspur Supreme' were intermediate.

Spur-type strains generally had a greater leaf area/spur than standard strains. At Clarksville, the leaf area/spur for spur types ranged from 110 ('Starkspur Ultrared') to $77 \mathrm{~cm}^{2}$ ('Starkspur Supreme'), while standard types, except 'Nured Royal' $\left(95 \mathrm{~cm}^{2}\right)$, had values ranging from 84 ('Classic Red') to $65 \mathrm{~cm}^{2}$ ('Improved Ryan Red'). At Kearneysville (Table 3), spur-types ranged from 105 ('ACN 501') to $74 \mathrm{~cm}^{2}$ ('Wellspur') and standard types, except 'Aomori' $\left(80 \mathrm{~cm}^{2}\right)$, from 73 ('Nured Royal') to $57 \mathrm{~cm}^{2}$ ('Improved Ryanred').

Leaf area per leaf. Differences in leaf area per spur among strains arose from differences in both leaf number per spur and leaf area per leaf. Within each growth type, some strains had high values for both leaf number and area, including 'ACN 501', 'Ace', and 'Sturdeespur' (Clarksville only), while others, such as 'Early Red One' and 'Improved Ryanred', had low values for both characters.

Specific leaf weight. The specific leaf weight of spur leaves did not vary markedly among strains at either of the two sites (Tables 2 and 3). However, spur-type strains generally had higher specific leaf weights than standard strains, the separation between types being most apparent at Kearneysville. Although the ranking of strains was quite different between the two sites, some strains were consistent in their placement; the specific leaf weights of 'Starkspur Supreme' and 'Sturdeespur' were high at both sites, while those of 'Sharp Red' and 'Topred' were consistently low.

Bud diameter. Differences in spur bud diameter among strains were small, and variability within each sample was considera- ble. However, spur-type strains generally had larger bud diameters than standard types. The smaller bud diameters at Clarksville (Table 2) than at Kearneysville were probably a consequence of the earlier sampling date.

Yield efficiency. Spur-type strains generally had a higher yield efficiency (i.e., yield/trunk cross-sectional area) at both sites. At Clarksville, the spur types 'Improved Ryan Spur', 'Atwood', 'Ace', 'Sturdeespur', and 'Apex' had high yields and high yield efficiencies, and the standard strains 'Aomori' and 'Sharp Red' had low values (Table 4). However, the standard strain 'Early Red One' had both the highest yield and yield efficiency. Individual mean fruit weight was largely unrelated to strain type or tree yield. At Kearneysville, the spur types 'Sturdeespur', 'Ace', and 'Redspur' from the 1981 planting and 'Cascade Spur' from the 1982 planting had the highest yields and the highest yield efficiencies (Table 5). The standard strains 'Triple Red', 'Sharp Red', and 'Aomori' had low values. Two standard strains, 'Imperial' and 'Improved Ryan Red', had yield efficiencies similar to those in the midrange for spur types and, perhaps because of the younger trees, 'Redchief' had a low yield efficiency when compared with other spur types.

Many of the different indices that were measured to determine spur quality in this study were closely and positively interrelated (Tables 6 and 7). Spur bud diameter was significantly correlated with spur leaf number, spur leaf area (Fig. 1), and leaf area per leaf, as was spur leaf number to area. Similarly, spur density was generally significantly correlated with bud diameter, spur leaf number, and spur leaf area (Fig. 2). Although many of the correlation coefficients were small, there was no indication that high spur densities were associated with a decline in quality of other spur attributes. At both sites in our study, spur density was negatively correlated with trunk cross-sectional area (Fig. 
Table 4. Trunk cross-sectional area (TCSA), fruit size, and yield of 28 'Delicious' apple strains growing at the Michigan State Univ. Clarksville Horticultural Research Station. ${ }^{\mathrm{P}}$

\begin{tabular}{lllccc}
\hline \hline \multicolumn{1}{c}{$\begin{array}{c}\text { Delicious } \\
\text { strain }\end{array}$} & Type & $\begin{array}{c}\text { TCSA } \\
\left(\mathrm{cm}^{2}\right)\end{array}$ & $\begin{array}{c}\text { Wt/fruit } \\
(\mathrm{g})\end{array}$ & $\begin{array}{c}\text { Yield/tree } \\
(\mathrm{kg})\end{array}$ & $\begin{array}{c}\text { Yield/TCSA } \\
\left(\mathrm{kg} \cdot \mathrm{cm}^{-2}\right)\end{array}$ \\
\hline Hardi-Brite Spur & Spur & 30.6 & 191 & 23.6 & 0.77 \\
Improved Ryan Spur & Spur & 40.8 & 203 & $\mathbf{3 7 . 9}$ & 0.93 \\
Red King Oregon Spur & Spur. & 37.4 & 207 & 29.0 & 0.79 \\
Starkrimson & Spur & 38.4 & 179 & 22.6 & 0.62 \\
Spured Royal & Spur & 32.1 & $\mathbf{1 6 4}$ & 26.5 & 0.85 \\
Redchief & Spur & 43.9 & $\mathbf{2 0 7}$ & 23.3 & 0.51 \\
Hardispur & Spur & 36.0 & 196 & 27.3 & 0.76 \\
Redspur & Spur & 40.9 & 182 & 27.0 & 0.65 \\
Silverspur & Spur & 42.0 & 174 & 29.2 & 0.71 \\
Ace & Spur & 38.5 & 188 & 34.5 & 0.92 \\
Sturdeespur & Spur & 41.3 & 183 & & 0.83 \\
Apex & Spur & 38.5 & 188 & 31.8 & 0.83 \\
Starkspur Supreme & Spur & 42.6 & & 31.5 & 0.72 \\
Wellspur & Spur & $\mathbf{4 1 . 6}$ & $\mathbf{1 7 5}$ & $\mathbf{2 6 . 4}$ & $\mathbf{0 . 6 5}$ \\
Wellspur & Spur & $\mathbf{3 4 . 6}$ & $\mathbf{1 7 5}$ & $\mathbf{3 3 . 1}$ & $\mathbf{0 . 9 5}$ \\
Starkspur Ultrared & Spur & 33.1 & 198 & 11.2 & 0.39 \\
Improved Ryanred & Standard & 5.6 & 201 & 28.5 & 0.54 \\
Imperial & Standard & 47.7 & 205 & 28.0 & 0.57 \\
Early Red One & Standard & $\mathbf{4 0 . 3}$ & $\mathbf{1 8 0}$ & $\mathbf{4 4 . 2}$ & $\mathbf{1 . 0 9}$ \\
August Red & Standard & 55.1 & 206 & 30.3 & 0.56 \\
Nured Royal & Standard & 62.8 & 200 & 20.6 & 0.32 \\
Starking (Mood) & Standard & $\mathbf{6 4 . 2}$ & $\mathbf{2 1 2}$ & $\mathbf{2 8 . 9}$ & $\mathbf{0 . 4 8}$ \\
Rose Red & Standard & $\mathbf{5 9 . 2}$ & $\mathbf{2 1 0}$ & $\mathbf{4 3 . 9}$ & $\mathbf{0 . 8 0}$ \\
Classic Red & Standard & 62.3 & $\mathbf{2 0 6}$ & 29.9 & 0.48 \\
Aomori & Standard. & $\mathbf{5 7 . 5}$ & $\mathbf{2 0 1}$ & 15.3 & 0.27 \\
Topred & Standard & 59.0 & $\mathbf{2 0 2}$ & 23.1 & 0.38 \\
Hi-Early & Standard & 60.0 & $\mathbf{2 1 3}$ & 20.3 & 0.35 \\
Sharp Red & Standard & 51.9 & 149 & 14.2 & 0.27 \\
LSD (P <0.05) & & 12.7 & 28.9 & 11.2 & 0.45 \\
\hline Darda & & & &
\end{tabular}

${ }^{\mathrm{z}}$ Data are means of six replications.

Table 5. Trunk cross-sectional area (TCSA) and yield of 23 'Delicious' apple strains growing at the West Virginia Univ. Experiment Farm, Kearneysvlle. ${ }^{\mathrm{z}}$

\begin{tabular}{llrrr}
\hline \multicolumn{1}{c}{$\begin{array}{c}\text { Delicious } \\
\text { strain }\end{array}$} & \multicolumn{1}{c}{ Type } & $\begin{array}{r}\text { TCSA } \\
\left(\mathrm{cm}^{2}\right)\end{array}$ & $\begin{array}{r}\text { Yield/tree } \\
(\mathrm{kg})\end{array}$ & $\begin{array}{r}\text { Yield/TCSA } \\
\left(\mathrm{kg} \cdot \mathrm{cm}^{-2}\right)\end{array}$ \\
\hline Sturdeespur & Spur & 17.9 & 20.1 & 1.13 \\
Redchief & Spur & 7.3 & $\mathbf{2 . 4}$ & 0.32 \\
Starkspur UltraStripe & Spur & $\mathbf{5 . 4}$ & 3.8 & 0.69 \\
Redspur & Spur & 21.0 & 19.0 & 0.92 \\
Ace & Spur & 15.0 & 17.6 & 1.27 \\
Imperial & Standard & 24.6 & 22.4 & 0.87 \\
Apex & Spur & 17.1 & 12.4 & 0.76 \\
Starkspur Supreme & Spur & 20.4 & 9.0 & 0.55 \\
Cascade Spur & Spur & 15.8 & 17.7 & 1.11 \\
ACN 501 & Spur & 5.0 & 3.3 & 0.69 \\
Starkspur Ultrared & Spur & 16.7 & 8.1 & 0.47 \\
Ryanred Spur & Spur & 17.5 & 13.8 & 0.82 \\
Starkrimson & Spur & 35.7 & 11.3 & 0.31 \\
Improved Ryanred & Standard & 22.4 & 15.7 & 0.70 \\
Wellspur & Standard & 26.3 & 12.4 & 0.47 \\
Triple Red & Standard & $\mathbf{2 4 . 5}$ & 7.6 & 0.31 \\
Classic Red & Standard & 29.9 & 11.4 & 0.38 \\
Red Prince & Standard & 34.6 & 12.4 & 0.37 \\
Topspur & Spur & $\overline{5}$ & $\overline{5.7}$ & $\mathbf{0 . 2 6}$ \\
Aomori & Standard & 24.5 & 8.1 & $\mathbf{0 . 3 5}$ \\
Topred & Standard & 23.7 & 4.7 & 0.16 \\
Sharp Red & Standard & 29.6 & 11.4 & 0.43 \\
Nured Royal & Standard & 26.2 & 8.7 & 0.46 \\
LSD (P < 0.05) & & 8.1 & & \\
\hline Data & & & &
\end{tabular}

${ }^{\mathrm{z}}$ Data are means of two blocks, each containing five trees. 
Table 6. Correlation coefficients between spur characteristics and yield indices of 'Delicious' strains growing at the Michigan State Univ. Clarksville Horticultural Research Station.

\begin{tabular}{|c|c|c|c|c|c|c|c|c|c|}
\hline $\begin{array}{l}\text { Spur and yield } \\
\text { characteristics }\end{array}$ & $\begin{array}{l}\text { Bud } \\
\text { diam }\end{array}$ & $\begin{array}{c}\text { Leaf } \\
\text { no./spur }\end{array}$ & $\begin{array}{c}\text { Leaf } \\
\text { area/spur }\end{array}$ & $\begin{array}{l}\text { Specific } \\
\text { leaf wt }\end{array}$ & $\begin{array}{c}\text { Leaf } \\
\text { area/leaf }\end{array}$ & $\begin{array}{c}\text { Spur } \\
\text { density }\end{array}$ & $\begin{array}{l}\text { Trunk cross- } \\
\text { sectional } \\
\text { area (TCSA) }\end{array}$ & $\mathrm{Wt} /$ fruit & Yield/tree \\
\hline Leaf no./spur & $0.26 * *$ & & & & & & & & \\
\hline Specific leaf wt & 0.07 & 0.04 & $-0.21 *$ & & & & & & \\
\hline Leaf area/leaf & $0.49^{* *}$ & 0.08 & $0.87 * *$ & $-039 * *$ & & & & & \\
\hline Spur density & $0.30 * *$ & $0.39 * *$ & $0.35 *$ & -0.07 & 0.20 & & & & \\
\hline Yield/tree & 0.15 & 0.01 & 0.00 & 0.14 & -0.06 & 0.12 & 0.07 & 0.00 & \\
\hline Yield efficiency & $0.19 *$ & 0.14 & 0.08 & 0.08 & -0.01 & $0.23 * *$ & $-0.55 * *$ & $-0.64 * *$ & $0.36^{* *}$ \\
\hline
\end{tabular}

****Significant at $P=0.05$ or 0.01 , respectively.

Table 7. Correlation coefficients between spur characteristics and yield indices of 'Delicious' strains growing at the West Virginia Univ. Experiment Farm, Kearneysville.

\begin{tabular}{|c|c|c|c|c|c|c|c|c|}
\hline $\begin{array}{l}\text { Spur and yield } \\
\text { characteristics }\end{array}$ & $\begin{array}{l}\text { Bud } \\
\text { diam }\end{array}$ & $\begin{array}{c}\text { Leaf } \\
\text { no./spur }\end{array}$ & $\begin{array}{c}\text { Leaf } \\
\text { area/spur }\end{array}$ & $\begin{array}{l}\text { Specific } \\
\text { leaf wt }\end{array}$ & $\begin{array}{c}\text { Leaf } \\
\text { area/leaf }\end{array}$ & $\begin{array}{c}\text { Spur } \\
\text { density }\end{array}$ & $\begin{array}{l}\text { Trunk cross- } \\
\text { sectional } \\
\text { area (TCSA) }\end{array}$ & Yield/tree \\
\hline Leaf no./spur & $0.52 * *$ & & & & & & & \\
\hline Leaf area/spur & $0.66 * *$ & $0.23 * *$ & & & & & & \\
\hline Specific leaf w-t & 0.16 & $0.66 * *$ & $0.49^{*}$ & & & & & \\
\hline Leaf area/leaf & $0.50 * *$ & 0.19 & $0.78 * *$ & 0.04 & & & & \\
\hline Spur density & 0.37 & $0.72 * *$ & $0.45^{*}$ & 0.30 & -0.02 & & & \\
\hline TCSA & $-0.46^{*}$ & $-0.71 * *$ & $-0.62 * *$ & -0.35 & -0.28 & $-0.65 * *$ & & \\
\hline Yield/tree & 0.08 & 0.14 & -0.06 & 0.08 & -0.35 & 0.33 & 0.17 & \\
\hline Yield efficiency & $0.47 *$ & $0.57 * *$ & $0.39 *$ & 0.36 & -0.04 & $0.66 * *$ & $-0.43 *$ & $0.76^{* *}$ \\
\hline
\end{tabular}

****Significant at $P=0.05$ or 0.01 , respectively.

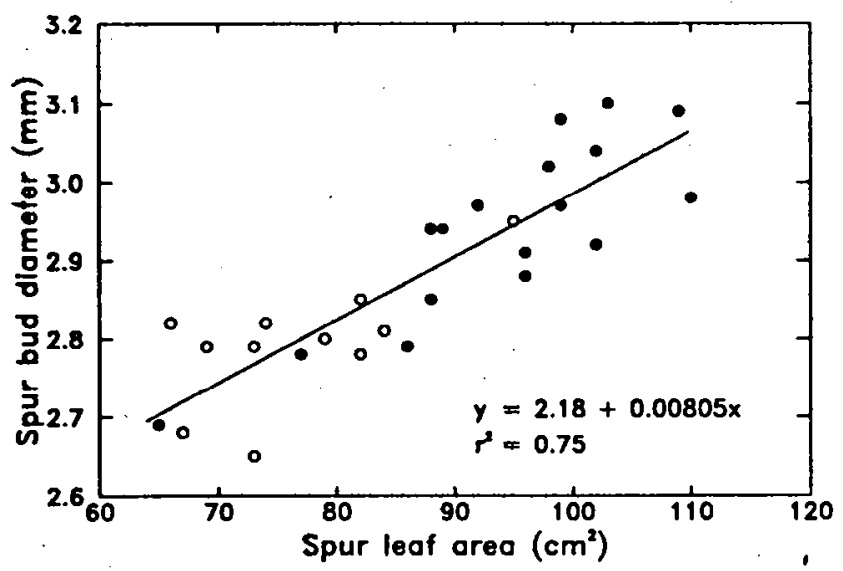

Fig. 1. Relationship between spur leaf area and terminal bud diameter for 28 'Delicious' strains growing at Clarksville, Mich. ( $\mathbf{O}$, spurtype strains; $\mathrm{O}$, standard strains). Data are means of six observations.

3), but positively correlated to yield efficiency (Fig. 4), indicating the value of this index in determining differences in tree performance (i.e., efficiency) among strains. In contrast, no spur characters were related to tree yield. Overall, therefore, spur-type strains had a higher density of spurs per unit limb length and were more vigorous with respect to attributes, such as spur leaf area, leaf number, and terminal bud diameter.

Rootstock effects. Rootstock had a considerable influence on the vegetative characteristics of the 'Starkspur Supreme' spurs. The number of leaves per spur, leaf area per spur, and leaf area per leaf were highest on MAC 24 and lowest on the dwarfing rootstocks MAC 9, M.9, and M.27 EMLA (Table 8). Scion

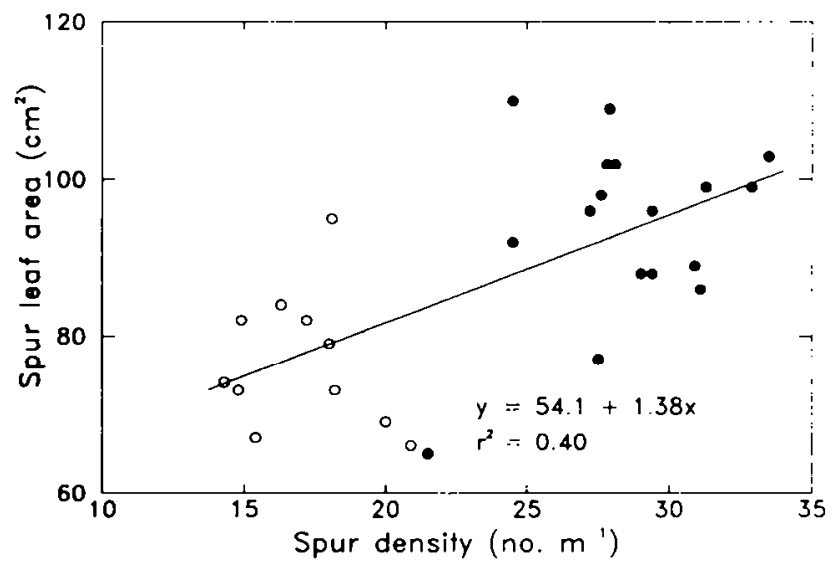

Fig. 2. Relationship between spur density and spur leaf area for 28 'Delicious' strains growing at Clarksville, Mich. ( $\mathbf{O}$, spur-type strains; $O$, standard strains). Data are means of six observations.

vigor (trunk cross-sectional area) values showed very similar trends (Table 9). In contrast, specific leaf weight was least for the most vigorous rootstocks-MAC 24, OAR 1, and M.7 EMLA (Table 8).

Spur density differences were small. However, MAC 9, one of the least vigorous dwarfing rootstocks, had the highest density (33 spurs $/ \mathrm{m}$ ), and MAC 24 , one of the most vigorous, the lowest density (19 spurs/m). The spur density on all other rootstocks was between 22 and 29 spurs $/ \mathrm{m}$.

Cumulative yield was highest for the most vigorous rootstock, MAC 24, and lowest for the least, M.27 EMLA. Other rootstocks followed a similar general relationship, except for OAR 


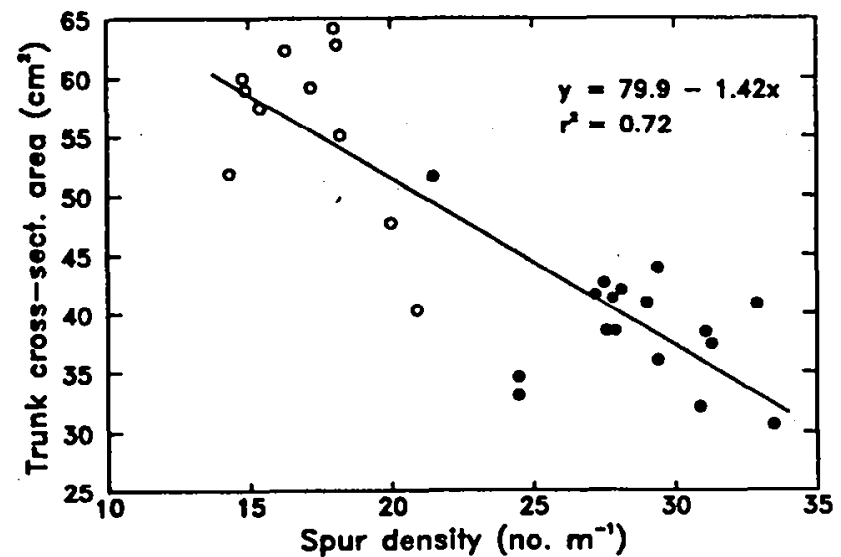

Fig. 3. Relationship between spur density and trunk cress-sectional area for 28 'Delicious' strains growing at Clarksville, Mich. ( $\mathbf{0}$, spurtype strains; $\bigcirc$, standard strains). Data are means of six observations.

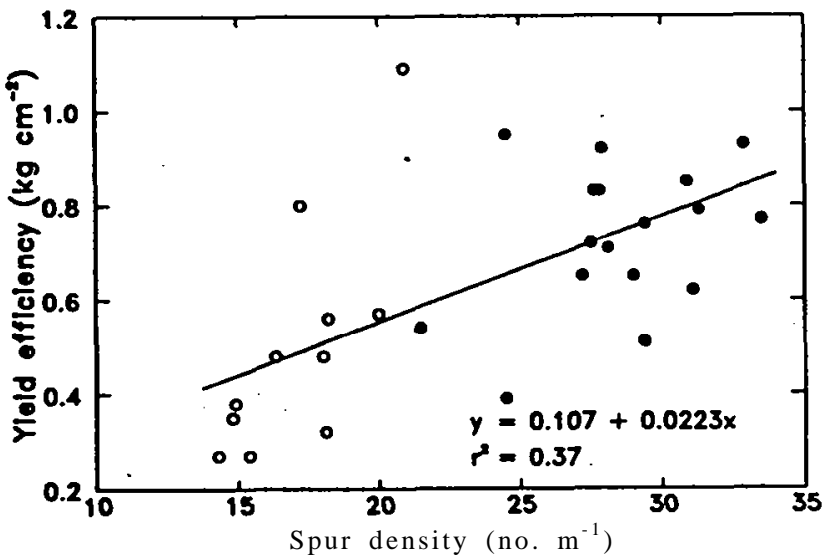

Fig. 4. Relationship between spur density and yield efficiency (i.e., yield/trunk cross-sectional area) for 28 'Delicious' strains growing at Clarksville, Mich. ( $\bullet$, spur-type strains; $\bigcirc$, standard strains). Data are means of six observations.

1, which had a low cumulative yield. This rootstock also had the lowest yield efficiency, while MAC 9, M.9, and M.9 EMLA had the highest (Table 9). When vegetative growth and yield of 'Starkspur Supreme' on the two closely related rootstocks M.9 and M.9 EMLA were compared, few of the differences between the two rootstocks were statistically significant. However, some indices of spur (leaf number and leaf area per spur) and tree
(TCSA) vigor, together with cumulative yield, were higher with the virus-free M.9 EMLA.

\section{Discussion}

Scion effects. Previous evaluations of 'Delicious' strains and selections have focused primarily on classifying fruit quality and yield characteristics. New strains of 'Delicious' are frequently introduced to the apple industry and the promotion of these strains is largely based on the fruit color and on the timing and nature (stripe or blush) of color development (Crassweller et al., 1985). Comparisons have also been made of fruit maturity among various strains. Strains are also promoted because of their spur habit, but classification of a strain into a spur, semispur, or standard type has previously been qualitative rather than quantitative.

Ketchie (1984) assessed 17 strains of 'Delicious' on seedling rootstock at Columbia, Wash., and found that spur types had a significantly higher spur density than standard types. However, some spur types, such as 'Sturdeespur' and 'Earlistripe', had similar spur densities as some standard types, such as 'Ryan', 'Imperial', 'Chelan Red', and 'Red King'. Spur density (all per meter length) ranged from 32 to 42 for spur types and from 25 to 30 for standard types and was stable from year to year. These values compare with 24 to 39 and 14 to $25 \mathrm{spurs} / \mathrm{m}$, respectively, in our study. Specific comparisons of some individual strains are possible. The respective values at Columbia, Clarksville, and Kearneysville were 40, 27, and 24 for 'Wellspur'; 39,29 , and 33 for 'Redspur'; 36, 28, and 39 for 'Sturdeespur'; 30, 20, and 32 for 'Imperial'; and 25, 14, and 16 for 'Sharp Red'. Factors such as site, rootstock, and cropping clearly influence the values obtained, and this variability may prevent classification on the basis of absolute spur density, as proposed by Ketchie (1984). However, classification on a relative basis appears possible, particularly as the ranking of strains between sites appears to be consistent within rootstock.

A characteristic feature of spur-type strains of 'Delicious' apple is their more-compact growth form and associated upright branch habit, compared with the more-open canopy form of standard strains. Consequently, spur-type strains can be planted closer together to maximize canopy development per unit land area. Assessments of productivity, therefore, must be made on the basis of both unit-tree production and unit-area production, and/or yield efficiency (i.e., yield per unit trunk cross-sectional area). Spur-type strains are frequently more productive than standard types, at least during the first 15 years of production. In a comparison of 28 strains growing at Wenatchee, Wash., in

Table 8. Influence of nine rootstocks on spur quality of 'Starkspur Supreme Delicious' (Ohio Agricultural Research and Development Center, 1986).

\begin{tabular}{lccccc}
\hline \hline & $\begin{array}{c}\text { Leaf } \\
\text { no./spur }\end{array}$ & $\begin{array}{c}\text { Leaf } \\
\text { area/spur } \\
\left(\mathrm{cm}^{2}\right)\end{array}$ & $\begin{array}{c}\text { Leaf } \\
\text { area/leaf } \\
\left(\mathrm{cm}^{2}\right)\end{array}$ & $\begin{array}{c}\text { specific } \\
\text { leaf wt } \\
\left(\mathrm{mg} \cdot \mathrm{cm}^{-2}\right)\end{array}$ & $\begin{array}{c}\text { Bud } \\
\text { diam } \\
(\mathrm{mm})\end{array}$ \\
\hline Ottawa 3 & 7.3 & 67.5 & 9.3 & 14.6 & 3.4 \\
M.7 EMLA & 6.9 & 78.0 & 11.2 & 12.5 & 3.6 \\
M.9 EMLA & 7.4 & 64.0 & 8.7 & 13.1 & 3.5 \\
M.26 EMLA & 6.9 & 65.5 & 9.5 & 13.2 & 3.6 \\
M.27 EMLA & 6.7 & 53.4 & 7.9 & 13.8 & 3.6 \\
M.9 & 6.2 & 56.6 & 9.0 & 12.8 & 3.8 \\
MAC 9 & 6.7 & 57.7 & 8.4 & 13.0 & 3.2 \\
MAC 24 & 7.5 & 86.6 & 11.6 & 10.8 & 3.3 \\
OAR 1 & 7.4 & 73.4 & 9.9 & 11.9 & 3.3 \\
LSD P $<0.05$ & $\mathbf{0 . 7}$ & 9.9 & 1.1 & 1.7 & NS \\
\hline
\end{tabular}

${ }^{\mathrm{z}}$ Data are means of 10 observations. 
Table 9. Influence of nine rootstocks on tree size and yield of 'Starkspur Supreme Delicious' (Ohio Agricultural Research and Development Center, 1986). ${ }^{\mathrm{z}}$

\begin{tabular}{lcccc}
\hline \hline & $\begin{array}{c}\text { Trunk cross- } \\
\text { sectional } \\
\left(\mathrm{cm}^{2}\right)\end{array}$ & $\begin{array}{c}\text { Yield/ } \\
\text { tree } \\
(\mathrm{kg})\end{array}$ & $\begin{array}{c}\text { Yield } \\
\text { efficiency } \\
\left(\mathrm{kg} \cdot \mathrm{cm}^{-2}\right)\end{array}$ & $\begin{array}{c}\text { Cumulative } \\
\text { yield } \\
(\mathrm{kg})\end{array}$ \\
\hline Ottawa 3 & 29.0 & 28.0 & 0.86 & 72.9 \\
M.7 EMLA & 47.3 & 41.2 & 0.82 & 79.0 \\
M.9 EMLA & $\mathbf{2 9 . 0}$ & 31.0 & $\mathbf{1 . 0 9}$ & 72.9 \\
M.26 EMLA & $\mathbf{3 7 . 3}$ & 33.2 & $\mathbf{0 . 8 9}$ & 69.9 \\
M.27 EMLA & $\mathbf{8 . 1}$ & $\mathbf{4 . 2}$ & 0.30 & 12.5 \\
M.9 & $\mathbf{1 7 . 7}$ & $\mathbf{2 1 . 8}$ & 1.27 & 49.6 \\
MAC 9 & $\mathbf{2 3 . 7}$ & 29.7 & 1.47 & 64.2 \\
MAC 24 & $\mathbf{8 6 . 9}$ & 55.8 & 0.52 & 107.8 \\
OAR 1 & 47.7 & 26.2 & 0.44 & 40.3 \\
LSD $P<0.05$ & 13.8 & 14.9 & 0.54 & 31.1 \\
\hline
\end{tabular}

${ }^{\mathrm{z}}$ Data are means of 10 observations.

a planting identical with that used at Clarksville, Ketchie (1987) found that of the 16 spur-type strains evaluated on M.7 EMLA, 12 were among the 14 highest yielding, based on cumulative yield per tree. In contrast, Lord et al. (1980) found that cumulative yield per tree was higher for six out of seven standard strains than for the spur-type strains 'Sturdeespur' and 'Starkrimson'. This is a surprising result, considering that these same strains produced high yields in Wenatchee (Ketchie, 1987) and in the present study at both Clarksville and Kearneysville. However, yield efficiency was considerably higher for these two spur-type strains. Similarly, Rom and Ferree (1984) found that the standard-type 'Starking' had a higher cumulative yield, but a lower yield efficiency than 'Starkrimson'. Ferree et al. (1975) found that mean cumulative yields per tree, after the first 10 growing seasons, were higher for 13 standard-type strains than for four spur-type strains. However, when tree spacing was adjusted to $7 \times 4.5 \mathrm{~m}$ for standard types and $5.5 \times 2.7 \mathrm{~m}$ for spur types, calculated yields per unit land area for spur types were $70 \%$ higher than for standard types.

Many of the standard strains evaluated in this study originated as limb or whole-tree variants from the original 'Delicious' (Maas, 1970; Fisher and Ketchie, 1981). These include 'Topred', 'Red Prince', and 'Starking'. Others, such as 'August Red', 'Classic Red', 'Early Red One', 'Hi-Early', and 'Rose Red', all originated less directly from 'Starking'. Of the spur types, many have been selected from 'Starking' also, including 'Redspur', 'Starkrimson', 'Starkspur Supreme', 'Starkspur Ultrared', and 'Wellspur'. Other spur strains have arisen less directly-'Ace' was a selection from 'Red King Oregon Spur' and this strain was selected from the standard strain 'Red King' that arose from 'Starking'. 'Redchief can also be traced back to 'Starking' via 'Starkrimson'. Another spur strain, 'Spured Royal', arose from 'Delicious' via the standard strains 'Richared' and 'Royal Red'; this is the same background as the standard strain 'Nured Royal'. Hence, while several of the spur strains have been selected as improved types or variants from other spur strains, many have also been selected directly from standard strains. As the different strains have arisen through spontaneous mutations from a wide range of sources, a range rather than segregation of values for each vegetative growth character might be expected. This was evident from some of the characters where individual strains did not segregate into distinctly separated spur- or standard-type categories (e.g., Figs. 1, 3, and 4), while for other characters (Fig. 2), some segregation into two distinct populations appears to have occurred.
A density of 20 to $21 \mathrm{spurs} / \mathrm{m}$ appeared to separate strains into spur and standard types on the basis of current fruit nursery descriptions and classifications, with spur types tending to have a higher and standard types a lower density. While spur characters were not correlated with tree yield, spur density and bud diameter were highly correlated with yield efficiency at both sites, suggesting that strains can be identified in regard to their yield performance from some spur characteristics.

Rootstock effects. Between two parts of this study, a major difference was identified among the inter-relationships of the various spur quality attributes. Among rootstocks, spur density was negatively related to other vigor attributes, such as spur leaf number and spur leaf area, whereas, among strains, spur density was positively correlated with these factors (Figs. 1 and 2, Tables 6 and 7). The most dwarfing rootstocks, therefore, reduced both stem extension growth (as assessed by spur density) and the vigor of individual spurs. In rootstock breeding and evaluation research, scion performance characteristics that could quantitatively indicate the capacity of a rootstock to control tree size would be particularly useful, especially if they allowed effective selection of desired types at an early stage of a program (Cummins and Aldwinckle, 1983). Previous workers have attempted to use vegetative growth characters as indices of rootstock vigor, but usually have compared leaves and shoots of the rootstock rather than those of a common scion (e.g., Miller, 1977).

In this study, close associations existed between many of the characters measured to define spur quality of the scion and the degree to which a rootstock controlled the overall vigor of the scion. While these results were limited to only nine rootstocks, they did consistently distinguish the most vigorous from the most dwarfing types. These responses were sufficiently consistent to warrant the further evaluation of spur growth responses as screening indices for vigor in rootstock breeding research.

\section{Literature Cited}

Crassweller, R.M., J. Walder, and R.L. Shewfelt. 1985. Color evaluation of seventeen strains of 'Delicious'. Fruit Var. J. 39:21-24.

Cummins, J.N. and H.S. Aldwinckle. 1983. Breeding apple rootstocks, p. 294-394. In: J. Janick (ed.). Plant breeding reviews. AVI, Westport, Conn.

Dalbro, A.S. 1966. De smo blade hos aebteraeer (Spur leaves of apple trees). Horticultura 20:4-5.

Dozier, W.A., Jr., J.W. Knowles, W.A. Griffey, H.E. Burgess, E.L. Mayton, A.A. Powell, and J.A. McGuire. 1984. Evaluation of five non-spur strains of Red Delicious apples in Central Alabama. Alabama Agr. Expt. Sta. Bul. 557.

Ferree, D.C., C.A. Morrison, and L.C. Shew. 1975. Red Delicious apples....which strain is best? Ohio Rpt. 60(2):19-22.

Ferree, D.C. and J.W. Palmer. 1982. Effect of spur defoliation and ringing during bloom on fruiting, fruit mineral level, and net photosynthesis of 'Golden Delicious' apple. J. Amer. Soc. Hort. Sci. 107:1182-1186

Fisher, D.V. and D.O. Ketchie. 1981. Survey of literature on red strains of 'Delicious'. Washington State Univ. Coll. of Agr. Res. Ctr. Bul. 0898

Hansen, P. 1971. 14C Studies on apple trees. VII. The early seasonal growth in leaves, flowers and shoots as dependent upon current photosynthates and existing reserves. Physiol. Plant. 25:469-473.

Harley, C.P., J.R. Magness, M.P. Masure, L.A. Fletcher, and E.S. Degman. 1942. Investigation on the cause and control of biennial bearing of apple trees. USDA Tech. Bul. 792.

Ingle, M. 1972. Studies on the maturity and storage behavior of Red Delicious budsports. West Virginia Agr. Expt. Sta. Bul. 609T.

Ketchie, D.O. 1984. Flowering, spur formation and limb angles of Delicious apple strains. Fruit Var. J. 38:150-152. 
Ketchie, D.O. 1987. Delicious strain evaluation given at horticulture meeting. The Goodfruit Grower 38(2):8-11.

Lord, W.J., R.A. Damon, Jr., and J.F. Anderson. 1980. A comparison of tree size, productivity, and fruit quality of 'Delicious' strains. J. Amer. Soc. Hort. Sci. 105:883-887.

Maas, V. 1970. Delicious, p. 45-67. In: North American apples: Varieties, rootstocks, outlook. Michigan State Univ. Press, East Lansing.

Miller, S.R. 1977. Selection criteria in the seedling stage for predicting apple rootstock vigor. Can. J. Plant Sci. 57:667-674.
Rom, C.R. and D.C. Ferree. 1984. Spur leaf characteristics of nine apple cultivars. Fruit Var. J. 38:2-5.

Tukey, R.B. and J.K. Ballard. 1969a. Index of Delicious apple strains and selections. Washington State Univ. Coll. Agr. Coop. Ext. Serv. Bul. EM 3120.

Tukey, R.B. and J.K. Ballard. 1969b. Characteristics of Delicious apple strains and selections. Washington State Univ. Coll. Agr. Coop. Ext. Serv. Bul. EM 3124 